3

4

5

6

7

9 Corresponding Author: Li Yang; State Key Laboratory of Biotherapy and Cancer Center, West

China Hospital, Sichuan University, Chengdu 610041, People’s Republic of China;

$11 \quad$ Email: yl.tracy73@gmail.com

$12 \dagger$ Rui Zhang and Lin Tang contributed equally to this work.

\section{remodeling the tumor microenvironment}

\author{
Rui Zhang ${ }^{1} \uparrow$, Lin Tang ${ }^{1} \dagger$, Binyan Zhao ${ }^{1}$, Yaomei $\operatorname{Tian}^{1}$, Bailing Zhou ${ }^{1}$, Yandong Mu², Li Yang ${ }^{1 *}$
}

${ }^{1}$ State Key Laboratory of Biotherapy and Cancer Center, West China Hospital, Sichuan University, and Collaborative Innovation Center for Biotherapy, Chengdu, 610041, China.

${ }^{2}$ Stomatology Department, Sichuan Provincial People's Hospital, University of Electronic Science and Technology of China, Chengdu, 611731, People's Republic of China

13 

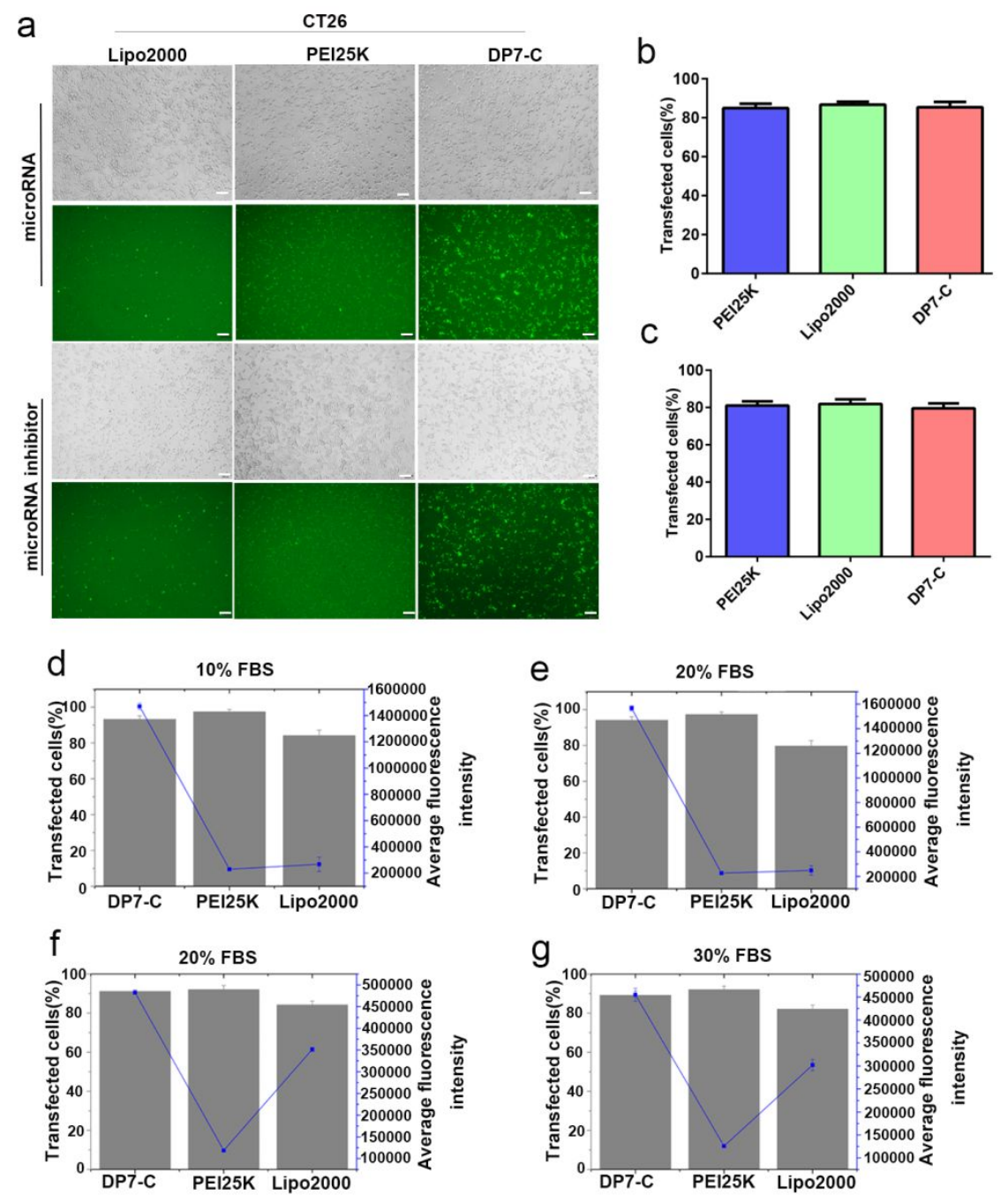

Figure S1. Efficiency of DP7-C transfection and serum stability of transfected microRNA or inhibitor. a-c. The efficiency of DP7-C-mediated transfection of microRNA and microRNA inhibitor to CT26. d-e. Detection of serum stability of DP7-C-transfected microRNA. f-g. Detection of serum stability of DP7-C-transfected microRNA inhibitor. Scale bar, $200 \mu \mathrm{m}$.

a

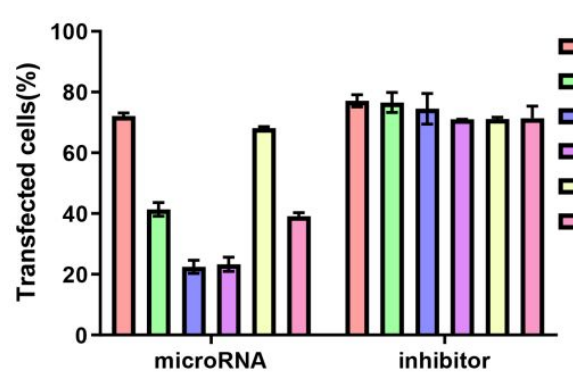

b

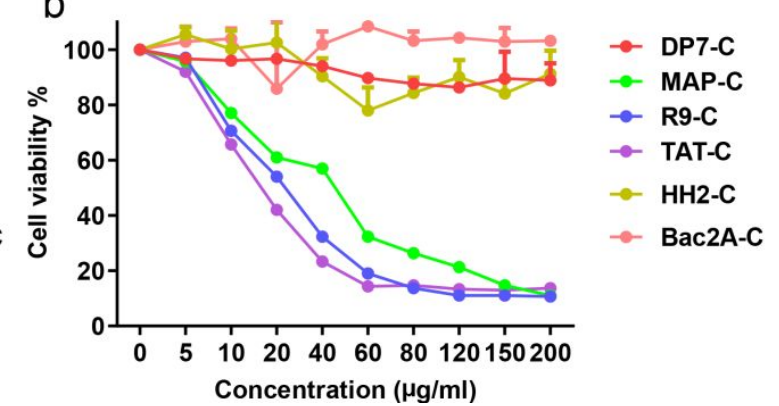

Figure S2. The transfection efficiency and the cytotoxicity of DP7-C, MAP-C, R9-C, TAT-C,

HH2-C and Bac2A-C. a. The transfection efficiency of DP7-C, MAP-C, R9-C, TAT-C, HH2-C and

Bac2A-C. b. The cytotoxicity of DP7-C, MAP-C, R9-C, TAT-C, HH2-C and Bac2A-C. 

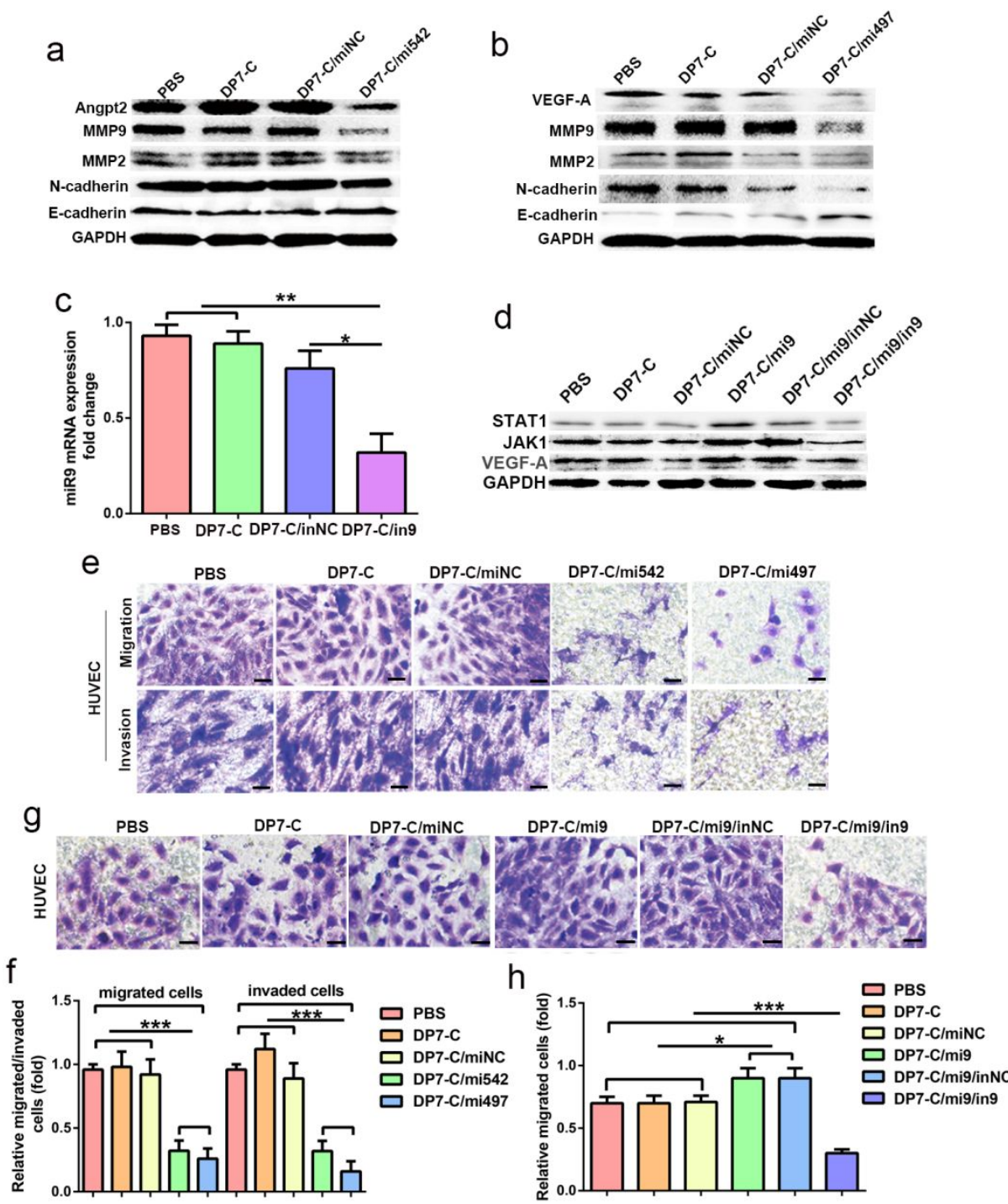

Figure S3. In vitro functional experiment. a. DP7-C-transfected mi542 inhibited the expression of mi542's target gene and its downstream signaling pathway-related proteins. b. DP7-C-mediated transfection of mi497 inhibited the expression of mi497's target gene and its downstream signaling pathway-related proteins. c. DP7-C-mediated transfection of in9 inhibited the expression of mi9 in 4T1 cells. d. DP7-C-mediated transfection of in9 inhibited the expression of mi9's target gene and its downstream signaling pathway-related proteins. e-h. Representative and quantitative results of transwell migration and invasion assays in HUVECs. Scale bar, $20 \mu \mathrm{m}$. 
a
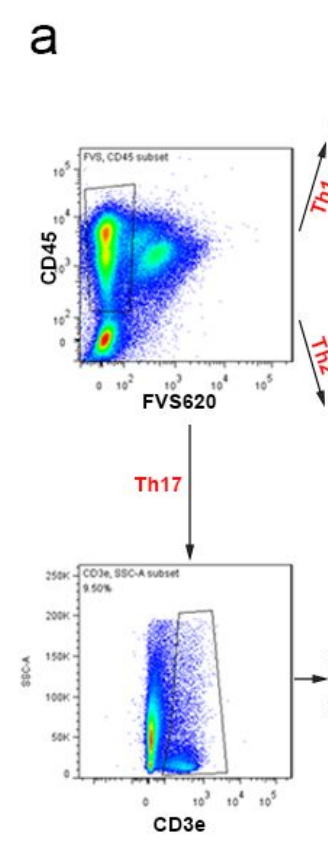
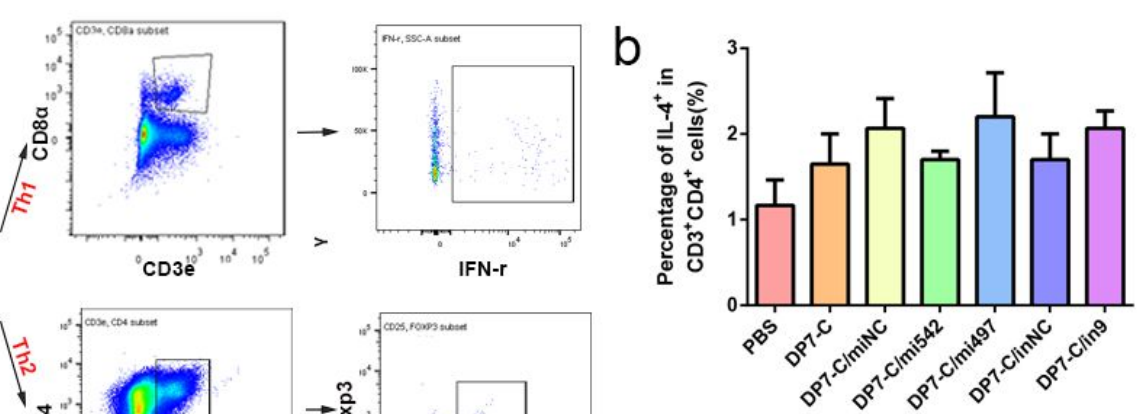

C

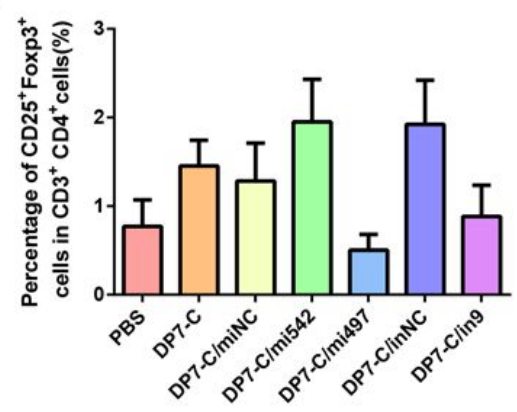

Figure S4. Detection of $\mathbf{T}$ cells in the tumor microenvironment. a. Representative cluster of $\mathrm{T}$ cell populations in the tumor microenvironment. b. Histogram of the percentage of Th2 cells. c. Histogram of the percentage of Tregs.

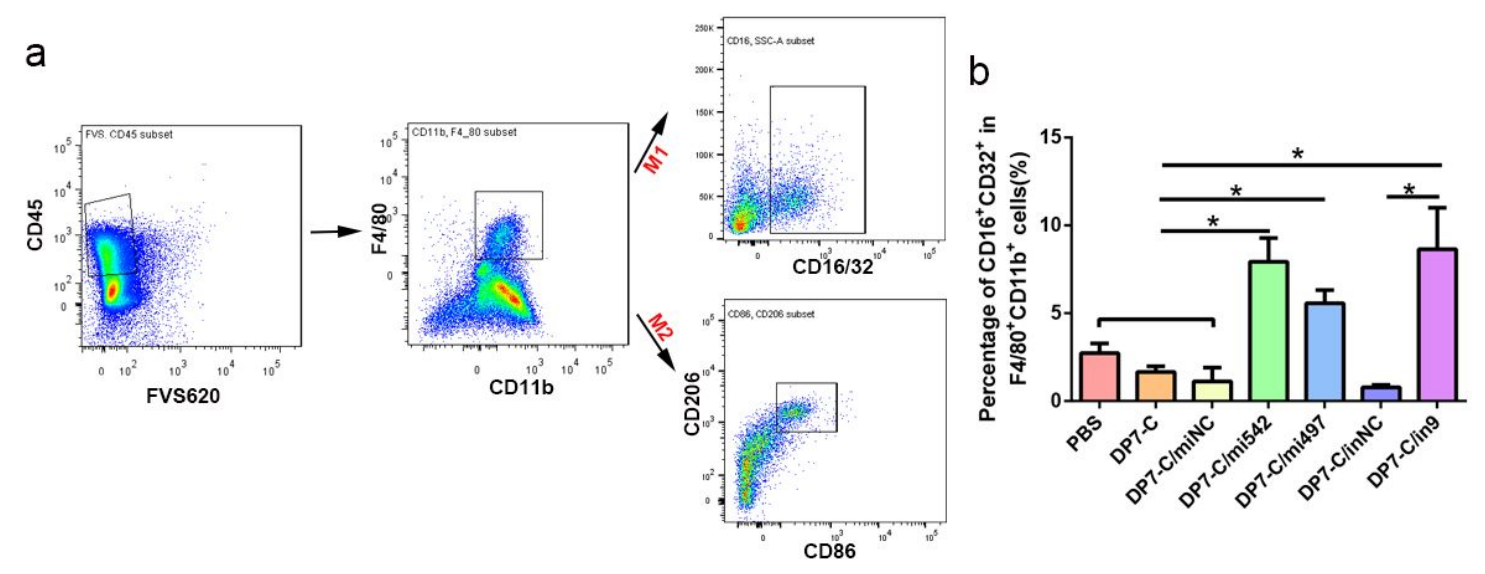

Figure S5. Detection of macrophages the tumor microenvironment. a. Representative cluster of macrophages in the tumor microenvironment. b. Histogram of M1 macrophage/M2 macrophages. * $p<0.05$. 

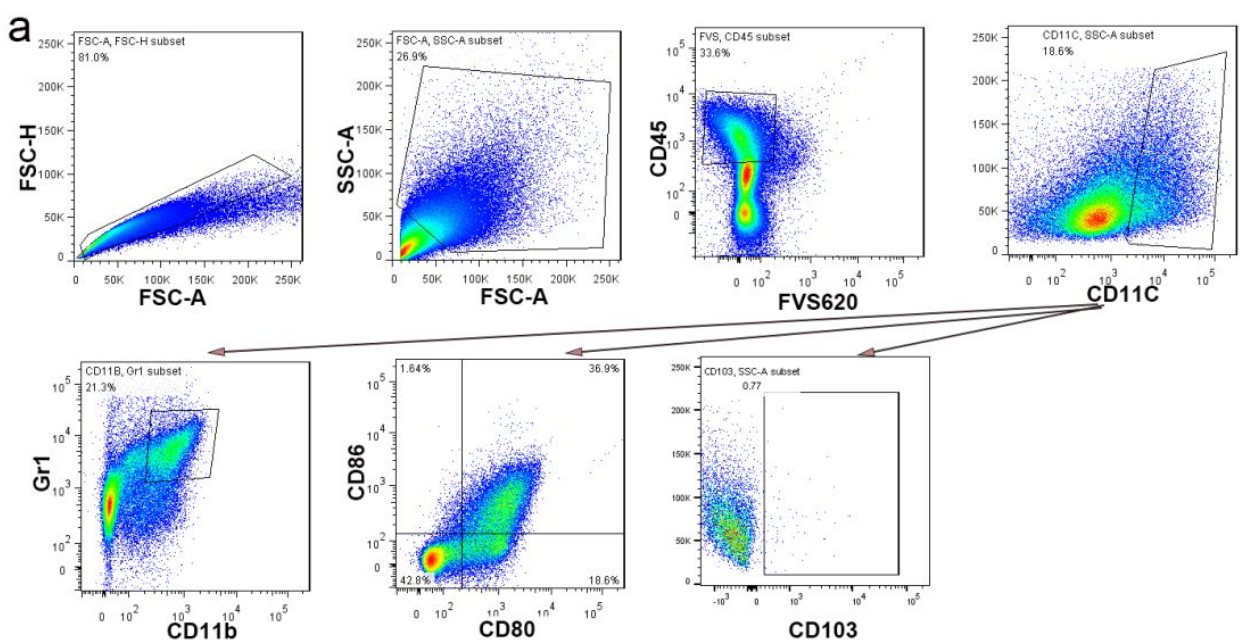

b

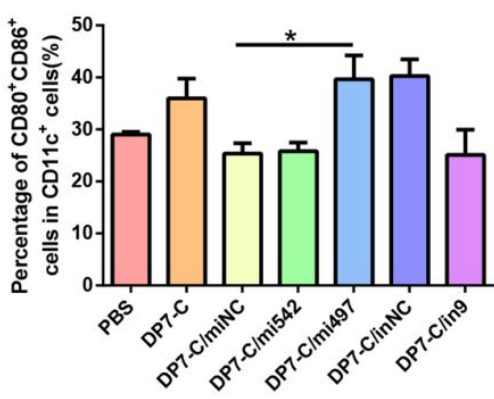

C

Figure S6. Detection of dendritic cells in the tumor microenvironment. a. Representative cluster of the DCs in the tumor microenvironment. b. Histogram of the percentage of mature DCs. c.

62 Histogram of mature DCs/MDSCs. ${ }^{*} p<0.05,{ }^{* *} p<0.01$ and ${ }^{* * *} p<0.001$. 